\title{
Backstepping Synthesis for Feedback Control of First-Order Hyperbolic PDEs with Spatial-Temporal Actuation
}

\author{
Xin Yu, ${ }^{1,2}$ Chao Xu, ${ }^{2}$ Huacheng Jiang, ${ }^{3}$ Arthi Ganesan, ${ }^{2}$ and Guojie Zheng ${ }^{4}$ \\ ${ }^{1}$ Laboratory of Information \& Control Technology, Ningbo Institute of Technology, Zhejiang University, Ningbo 315100, China \\ ${ }^{2}$ The State Key Laboratory of Industrial Control Technology and Institute of Cyber-Systems \& Control, Zhejiang University, \\ Hangzhou 310027, China \\ ${ }^{3}$ Department of Mathematics, Zhejiang University, Hangzhou 310027, China \\ ${ }^{4}$ College of Mathematics \& Information Science, Henan Normal University, Xinxiang 453007, China
}

Correspondence should be addressed to Chao Xu; cxu@zju.edu.cn

Received 27 March 2014; Revised 30 June 2014; Accepted 10 July 2014; Published 14 August 2014

Academic Editor: Milan Pokorny

Copyright (c) 2014 Xin Yu et al. This is an open access article distributed under the Creative Commons Attribution License, which permits unrestricted use, distribution, and reproduction in any medium, provided the original work is properly cited.

\begin{abstract}
This paper deals with the stabilization problem of first-order hyperbolic partial differential equations (PDEs) with spatial-temporal actuation over the full physical domains. We assume that the interior actuator can be decomposed into a product of spatial and temporal components, where the spatial component satisfies a specific ordinary differential equation (ODE). A Volterra integral transformation is used to convert the original system into a simple target system using the backstepping-like procedure. Unlike the classical backstepping techniques for boundary control problems of PDEs, the internal actuation can not eliminate the residual term that causes the instability of the open-loop system. Thus, an additional differential transformation is introduced to transfer the input from the interior of the domain onto the boundary. Then, a feedback control law is designed using the classic backstepping technique which can stabilize the first-order hyperbolic PDE system in a finite time, which can be proved by using the semigroup arguments. The effectiveness of the design is illustrated with some numerical simulations.
\end{abstract}

\section{Introduction}

Motivated by the essence of spatial-temporal evolutions in nature, control of systems governed by partial differential equations (PDEs), or distributed parameter system, has been studied for decades (e.g., [1-5], just to name a few). As one important category of distributed parameter systems, hyperbolic PDEs are widely used to describe dynamics arising in traffic flows, chemical reactors, heat exchangers, and fluid transport pipelines and the control problems regarding hyperbolic PDEs have been widely studied [6-9].

We consider the stabilization problem of a hyperbolic PDE system with a spatial-temporal actuation over the full physical domain. Our approach in this work relies on the backstepping method, which was originally developed in the 1990s for adaptive and robust control of nonlinear lumped parameter systems governed by ordinary differential equations (ODEs) [10]. In the last decade, the backstepping method for PDEs with boundary actuation has been widely developed [11-15]. Besides successful applicability of the backstepping techniques to one-dimensional classical PDE systems of both hyperbolic and parabolic types, much progress has been made to establish the control design for higher-dimensional systems, including complex PDEs arising in applied physics, including magnetohydrodynamic (MHD) [16] and fluid flows [17, 18] (governed by MHD and Navier-Stokes equations, resp.), pipeline dynamics for oil and gas transportation [19], and even 3-dimensional diffusion-reaction systems with varying parameters [20]. In addition, the backstepping technique can be extended to handle PDE systems with nonlinear terms in the sense of local stabilization $[21,22]$. 
Backstepping can be used to achieve the stabilization of unstable PDEs in a physically appealing way where the destabilizing terms are eliminated by means of an invertible integral transformation of the PDE together with the boundary feedback. In addition, unlike the linear quadratic regulator (LQR) approaches for boundary control [23, 24], which require the solutions of operator Riccati equations, the backstepping technique takes advantage of the structure of systems and yields control gain formulas which can be evaluated using symbolic computation and, in some cases, can be given explicitly. For detailed information on the backstepping method, one can refer to the books $[13,25]$ and the references therein.

The backstepping approach is so far a systematic method and gives a rather straightforward way for boundary feedback controller design for PDE systems. More recently, the authors in [26] have extended the backstepping method in order to deal with full domain control problems of parabolic PDEs. The authors first apply the backstepping transformation to map the original system into a simple target system. Then, an additional differential transformation is introduced to move the input to the boundary, and immediately an exponentially stabilizing state feedback controller is obtained.

The problem of boundary feedback stabilization of firstorder hyperbolic PDEs has been studied in [7] using the backstepping method. However, the backstepping technique has never been extended to deal with the full domainv control problem for the first-order hyperbolic PDE systems. In this paper, we assume that the interior actuator can be decomposed into a product of spatial and temporal components, and the spatial component satisfies a specific ODE such that the backstepping technique can be used to this problem. Following the backstepping procedure, in the first step, a simple system can be obtained from the original system by using the backstepping transformation. However, the input still remains in the obtained system for the interior actuation and we can not cancel the residual term using the classical backstepping technique, which causes the instability of the open-loop system. To solve this problem, a differential transformation is introduced to move the input from the interior of the domain to the boundary which enables removing the residual term [26]. Then, a feedback control law is obtained such that the first-order hyperbolic PDE converges to zero in a finite time, which has been proved using the semigroup argument. The main contribution of the current work is to extend the strategy proposed in [26] to first-order hyperbolic PDE systems.

The rest of this paper is organized as follows. In Section 2, we state the problem formulation. In Section 3, we discuss the state transformation to restate the control problem; then a differential transformation is given to transform the problem into a classical boundary stabilization problem. In Section 4, the invertibility of the proposed transformation is investigated. In Section 5, a state feedback controller is designed and the stability result is proved. The numerical simulations are presented in Section 6. In Section 7, we close the paper by addressing the concluding remarks and future research topics.

\section{Problem Formulation}

We consider the following 1-dimensional hyperbolic PDE of the form

$$
\begin{gathered}
w_{t}(x, t)=w_{x}(x, t)+\lambda(x) w(x, t)+h(x) w(0, t) \\
+\int_{0}^{x} f(x, y) w(y, t) d y+g(x) U(t), \\
(x, t) \in(0,1) \times(0, \infty), \\
w(1, t)=0, \quad t \in(0, \infty),
\end{gathered}
$$

where $w(x, t) \in \mathbb{R}$ is the state; $U(t) \in \mathbb{R}$ is the control input; $\lambda, h, g$ are continuous on $[0,1]$ and $f$ is continuous on $[0,1] \times[0,1]$. To use the backstepping technique to deal with the stabilization problem of (1), we make the following assumption on the function $g$ throughout of the paper, which represents the shape of actuation:

(A) $g$ satisfies the following integrodifferential equation:

$$
\begin{aligned}
& g^{\prime}(x)+\lambda(x) g(x) \\
& =\gamma g(x)-\int_{0}^{x} f(x, y) g(y) d y-g_{0} h(x), \\
& g(0)=g_{0},
\end{aligned}
$$

where $\gamma, g_{0} \in \mathbb{R}$ are two design parameters.

Since (2) is linear, for given $\gamma, g_{0} \in \mathbb{R}$, there exists a unique solution to (2). In the following, we assume that

$$
g_{0} \neq 0 \text {. }
$$

Otherwise, by the uniqueness theory of the solution for ODEs, $g(x)=0$ for any $x \in[0,1]$ if $g_{0}=0$. The choosing methods of the parameters $\gamma$ and $g_{0}$ will be specified in the following sections. The main objective of this paper is to stabilize the zero equilibrium of the system (1) with a state feedback controller.

\section{Transformations}

In the following, the design procedure is presented to the state feedback controller for the system (1). By using an infinitedimensional backstepping transformation, the system (1) can be converted into a simple system. Then, since the obtained system is not a boundary control system, an additional differential transformation is used to move the actuation function to the boundary. Based on the invertibility of these transformations, we construct a feedback control law such that the first-order hyperbolic PDE converges to zero in a finite time.

3.1. State Transformation. We first introduce the following state transformation for system (1):

$$
u(x, t)=w(x, t)\left[\exp \int_{0}^{x} \lambda(\eta) d \eta\right]
$$


which results in the following system:

$$
\begin{aligned}
u_{t}(x, t)= & u_{x}(x, t)+\bar{h}(x) u(0, t) \\
+ & +\int_{0}^{x} \bar{f}(x, y) u(y, t) d y+\bar{g}(x) U(t), \\
& (x, t) \in(0,1) \times(0, \infty), \\
& u(1, t)=0, \quad t \in(0, \infty),
\end{aligned}
$$

where

$$
\begin{aligned}
\bar{h}(x) & =h(x) \exp \left[\int_{0}^{x} \lambda(\eta) d \eta\right], \\
\bar{g}(x) & =g(x) \exp \left[\int_{0}^{x} \lambda(\eta) d \eta\right], \\
\bar{f}(x, y) & =f(x, y) \exp \left[\int_{y}^{x} \lambda(\eta) d \eta\right] .
\end{aligned}
$$

It follows from (2) that $\bar{g}$ satisfies the following integrodifferential equation:

$$
\begin{gathered}
\bar{g}^{\prime}(x)=\gamma \bar{g}(x)-\int_{0}^{x} \bar{f}(x, y) \bar{g}(y) d y-g_{0} \bar{h}(x), \\
\bar{g}(0)=g_{0},
\end{gathered}
$$

where $g_{0}$ is given in (2).

3.2. Backstepping Transformation. Now, we apply the backstepping transformation

$$
v(x, t)=u(x, t)-\int_{0}^{x} k(x, y) u(y, t) d y
$$

to the system (5), where the integral kernel function $k$ is a solution of the following PDE:

$$
\begin{gathered}
k_{x}(x, y)+k_{y}(x, y)=\int_{y}^{x} k(x, \eta) \bar{f}(\eta, y) d \eta-\bar{f}(x, y), \\
(x, y) \in(0,1) \times(0,1), \\
k(x, 0)=\int_{0}^{x} k(x, y) \bar{h}(y) d y-\bar{h}(x) .
\end{gathered}
$$

By [7], (9) has a unique solution $k \in C^{1}([0,1] \times[0,1])$. Now, by taking the derivatives of (8) with respect to $t$ and $x$, respectively, we can obtain

$$
\begin{aligned}
v_{t}(x, t)= & u_{t}(x, t)-\int_{0}^{x} k(x, y) u_{t}(y, t) d y \\
= & u_{x}(x, t)+[\bar{h}(x)+k(x, 0) \\
& \left.\quad-\int_{0}^{x} k(x, y) \bar{h}(y) d y\right] u(0, t) \\
& +\int_{0}^{x}\left[\bar{f}(x, y)+k_{y}(x, y)\right. \\
& \left.\quad-\int_{y}^{x} k(x, \eta) \bar{f}(\eta, y) d \eta\right] u(y, t) d y \\
= & u_{x}(x, t) \\
& +\int_{0}^{x}\left[\bar{f}(x, y)+k_{y}(x, y)\right. \\
& \left.-\int_{y}^{x} k(x, \eta) \bar{f}(\eta, y) d \eta\right] u(y, t) d y \\
& -k(x, x) u(x, t)+G(x) U(t), \\
& -\int_{0}^{x} k_{x}(x, y) u(y, t) d y, \\
v_{x}(x, t)= & u_{x}(x, t)-k(x, x) u(x, t)
\end{aligned}
$$

where we have exchanged the order of the integration in (10) and

$$
G(x)=\bar{g}(x)-\int_{0}^{x} k(x, y) \bar{g}(y) d y .
$$

Here, we note that in fact $G$ is the image of $\bar{g}$ under the backstepping transformation. Subtracting (11) from (10) and making use of (9), we have

$$
v_{t}(x, t)=v_{x}(x, t)+G(x) U(t), \quad(x, t) \in(0,1) \times(0, \infty) .
$$


Moreover, by (7), (9), and (12), we have

$$
\begin{aligned}
G^{\prime}(x) & -\gamma G(x) \\
= & \bar{g}^{\prime}(x)-k(x, x) \bar{g}(x)-\int_{0}^{x} k_{x}(x, y) \bar{g}(y) d y \\
& -\gamma\left[\bar{g}(x)-\int_{0}^{x} k(x, y) \bar{g}(y) d y\right] \\
= & \bar{g}^{\prime}(x)-k(x, x) \bar{g}(x)+\int_{0}^{x} k_{y}(x, y) \bar{g}(y) d y \\
& -\int_{0}^{x} \bar{g}(y) \int_{y}^{x} k(x, \eta) \bar{f}(\eta, y) d \eta d y \\
& +\int_{0}^{x} \bar{f}(x, y) \bar{g}(y) d y \\
& -\gamma\left[\bar{g}(x)-\int_{0}^{x} k(x, y) \bar{g}(y) d y\right] \\
= & \bar{g}^{\prime}(x)-\gamma \bar{g}(x)+\int_{0}^{x} \bar{f}(x, y) \bar{g}(y) d y+g_{0} \bar{h}(x) \\
& -\gamma\left[\bar{g}(x)-\int_{0}^{x} k(x, y) \bar{g}(y) d y\right] \\
& +\int_{0}^{x} \bar{f}(x, y) \bar{g}(y) d y \\
& -\int_{0}^{x} k(x, y) \bar{f}(y)-\int_{0}^{x} k(x, y) \bar{g}(y) d y \\
& \int_{0}^{\prime}(y, \eta) \bar{g}(\eta) d \eta d y
\end{aligned}
$$$$
=0 \text {, }
$$

where the order of the integration in the above equation has been exchanged. Thus, it follows from (7) and (14) that $G$ is the solution of the following ODE:

$$
G^{\prime}(x)=\gamma G(x), \quad G(0)=g_{0} .
$$

Now, we will discuss the boundary condition that $v$ should satisfy. Motivated by [7], the transformation (8) with the integral kernel $k$ satisfying (9) is invertible and the inverse transformation is of the form

$$
u(x, t)=v(x, t)+\int_{0}^{x} l(x, y) v(y, t) d y
$$

where the integral kernel $l \in C^{1}([0,1] \times[0.1])$ is the solution of the following PDE:

$$
\begin{gathered}
l_{x}(x, y)+l_{y}(x, y)=-\int_{y}^{x} \bar{f}(x, \eta) l(\eta, y) d \eta-\bar{f}(x, y), \\
\quad(x, y) \in(0,1) \times(0,1), \\
l(x, 0)=-\bar{h}(x) .
\end{gathered}
$$

Since $u(1, t)=0$ for $t \in(0, \infty)$, it follows from (16) that

$$
v(1, t)+\int_{0}^{1} l(1, y) v(y, t) d y=0, \quad t \in(0, \infty) .
$$

By combining (13) and (18), the backstepping transformation converts the system (5) into the following system:

$$
\begin{array}{r}
v_{t}(x, t)=v_{x}(x, t)+G(x) U(t), \\
(x, t) \in(0,1) \times(0, \infty), \\
v(1, t)+\int_{0}^{1} l(1, y) v(y, t) d y=0, \\
t \in(0, \infty) .
\end{array}
$$

From (19), we see that the input $U$ still remains on the internal domain and we can not eliminate the residual term at the boundary, which causes the instability of the open-loop system.

3.3. Differential Transformation. We introduce the following differential transformation:

$$
\phi(x, t)=v_{x}(x, t)-\gamma v(x, t) .
$$

Assume that the solution $v$ of (19) is sufficiently smooth such that the continuous derivatives in the following reduction all exist. The regularity of the solution $v$ will be discussed in the proof of Theorem 4. Then, under this assumption, by (19), we have

$$
\begin{aligned}
v_{t}(x, t) & =v_{x}(x, t)+G(x) U(t) \\
& =\phi(x, t)+\gamma v(x, t)+G(x) U(t) .
\end{aligned}
$$

Taking the derivative of (20) with respect to $t$ and taking the derivative of (21) with respect to $x$, respectively, we have

$$
\begin{gathered}
\phi_{t}(x, t)=v_{x t}(x, t)-\gamma v_{t}(x, t), \\
v_{t x}(x, t)=\phi_{x}(x, t)+\gamma v_{x}(x, t)+G^{\prime}(x) U(t) .
\end{gathered}
$$

Substituting (21) and (23) into the right-hand side of (22) yields

$$
\begin{aligned}
\phi_{t}(x, t)= & \phi_{x}(x, t)+\gamma v_{x}(x, t)+G^{\prime}(x) U(t) \\
& -\gamma[\phi(x, t)+\gamma v(x, t)+G(x) U(t)] \\
= & \phi_{x}(x, t)+\left[G^{\prime}(x)-\gamma G(x)\right] U(t) \\
& +\gamma\left[v_{x}(x, t)-\phi(x, t)-\gamma v(x, t)\right] .
\end{aligned}
$$


Thus, together with (15) and (20), this implies that

$$
\phi_{t}(x, t)=\phi_{x}(x, t)
$$

On the other hand, taking the derivative of (18) with respect to $t$, we have

$$
\begin{aligned}
0= & v_{t}(1, t)+\int_{0}^{1} l(1, y) v_{t}(y, t) d y \\
= & \phi(1, t)+\gamma v(1, t)+G(1) U(t) \\
& +\int_{0}^{1} l(1, y)[\phi(y, t)+\gamma v(y, t)+G(y) U(t)] d y \\
= & \phi(1, t)+\int_{0}^{1} l(1, y) \phi(y, t) d y+\bar{g}(1) U(t),
\end{aligned}
$$

where we have used the following equation:

$$
\bar{g}(1)=G(1)+\int_{0}^{1} l(1, y) G(y) d y
$$

Thus, we see from (25) and (26) that when the solution $v$ of (19) is sufficiently smooth, the differential transformation (20) can map $v$ to the solution $\phi$ of the following system:

$$
\begin{gathered}
\phi_{t}(x, t)=\phi_{x}(x, t), \\
(x, t) \in(0,1) \times(0, \infty), \\
\phi(1, t)+\int_{0}^{1} l(1, y) \phi(y, t) d y=-\bar{g}(1) U(t), \\
t \in(0, \infty) .
\end{gathered}
$$

\section{Inverse Transformation}

In this section, we describe the invertibility of the differential transformation defined by (20). We define a linear operator $T$ in $L^{2}(0,1)$ as

$$
T v=v^{\prime}-\gamma v, \quad \forall v \in \mathscr{D}(T)
$$

where

$$
\mathscr{D}(T)=\left\{v \in H^{1}(0,1) \mid v(1)+\int_{0}^{1} l(1, y) v(y) d y=0\right\} .
$$

Obviously, $T$ is an unbounded operator in $L^{2}(0,1)$. To show the invertibility of the differential transformation (20), it is equivalent to show the invertibility of $T$. Moreover, we can obtain that the inverse operator of $T$ is continuous under certain condition. To this end, we define a continuous function $p$ by

$$
p(x)=e^{\gamma x}+\int_{0}^{x} l(x, y) e^{\gamma y} d y .
$$

Theorem 1. Assuming that $p(1) \neq 0$, then the operator $T$ has an inverse operator $T^{-1}$ in $L^{2}(0,1)$. Moreover, there exist constants $C_{1}$ and $C_{2}$ such that

$$
\left\|T^{-1} \phi\right\| \leq C_{1}\|\phi\|, \quad\left\|T^{-1} \phi\right\|_{1} \leq C_{2}\|\phi\|,
$$

for any $\phi \in L^{2}(0,1)$, where $\|\cdot\|$ and $\|\cdot\|_{1}$ denote the usual norms of $L^{2}(0,1)$ and $H^{1}(0,1)$, respectively.

Proof. To verify the existence of $T^{-1}$ in $L^{2}(0,1)$, we need to show that, for any $\phi \in L^{2}(0,1)$, there exists a unique function $v \in \mathscr{D}(T)$ such that $\phi=T v$. For any given $c \in \mathbb{R}$, the following $\operatorname{ODE} v^{\prime}-\gamma v=\phi$ has a unique solution:

$$
v(x)=c e^{\gamma x}+\int_{0}^{x} e^{\gamma(x-y)} \phi(y) d y,
$$

which satisfies $v(0)=c$. We can see easily $v, v^{\prime} \in L^{2}(0,1)$, that is, $v \in H^{1}(0,1)$. According to (29) and (30), we remain to show that the function $v$ satisfies the boundary condition

$$
v(1)+\int_{0}^{1} l(1, y) v(y) d y=0 .
$$

By substituting (33) into (34), we have

$$
\begin{aligned}
v(1) & +\int_{0}^{1} l(1, y) v(y) d y \\
= & c e^{\gamma}+\int_{0}^{1} e^{\gamma(1-y)} \phi(y) d y \\
& +\int_{0}^{1} l(1, y)\left[c e^{\gamma y}+\int_{0}^{y} e^{\gamma(y-\eta)} \phi(\eta) d \eta\right] d y \\
= & c\left[e^{\gamma}+\int_{0}^{1} l(1, y) e^{\gamma y} d y\right] \\
& +\int_{0}^{1} \phi(y)\left[e^{\gamma(1-y)}+\int_{y}^{1} l(1, \eta) e^{\gamma(\eta-y)} d \eta\right] d y \\
= & c p(1)+\int_{0}^{1} \phi(y) \Gamma(y) d y,
\end{aligned}
$$

where

$$
\Gamma(y)=e^{\gamma(1-y)}+\int_{y}^{1} l(1, \eta) e^{\gamma(\eta-y)} d \eta
$$

Thus, if $p(1) \neq 0$, we can choose

$$
c=\frac{-1}{p(1)} \int_{0}^{1} \phi(y) \Gamma(y) d y
$$

such that (34) holds; that is, $v$ satisfies the boundary condition. Thus, we have proved that the operator $T$ has an inverse operator $T^{-1}$.

Next, we will prove (32). Noting that the function $\Gamma$ is continuous on $[0,1]$, we have

$$
|c| \leq \frac{1}{|p(1)|} \max _{x \in[0,1]}|\Gamma(x)|\|\phi\| .
$$


Combining (33) and (38) yields that

$$
\begin{aligned}
\|v\| & \leq \max _{x \in[0,1]}\left|e^{\gamma x}\right||c|+\max _{x \in[0,1]}\left|e^{\gamma x}\right|\|\phi\| \\
& \leq\left(\frac{1}{|p(1)|} \max _{x \in[0,1]}|\Gamma(x)| \max _{x \in[0,1]}\left|e^{\gamma x}\right|+\max _{x \in[0,1]}\left|e^{\gamma x}\right|\right)\|\phi\| \\
& =C_{1}\|\phi\|,
\end{aligned}
$$

where $C_{1}=(1 /|p(1)|) \max _{x \in[0,1]}|\Gamma(x)| \max _{x \in[0,1]}\left|e^{\gamma x}\right|+$ $\max _{x \in[0,1]}\left|e^{\gamma x}\right|$. Thus, we have proved the first inequality of (32). Moreover, since

$$
\begin{aligned}
v^{\prime}(x) & =c \gamma e^{\gamma x}+\phi(x)+\gamma \int_{0}^{x} e^{\gamma(x-y)} \phi(y) d y \\
& =\gamma v(x)+\phi(x),
\end{aligned}
$$

it follows from (39) that

$$
\left\|v^{\prime}\right\| \leq\left(\gamma C_{1}+1\right)\|\phi\| .
$$

Together with (39) again, this implies that there exists a constant $C_{2}$ such that $\|v\|_{1} \leq C_{2}\|\phi\|$; that is,

$$
\left\|T^{-1} \phi\right\|_{1} \leq C_{2}\|\phi\| \text {. }
$$

Thus the proof is complete.

Proposition 2. $p(1) \neq 0$ if and only if $\bar{g}(1) \neq 0$, where $p$ is defined by (31) and $\bar{g}$ is the solution of (7).

Proof. From (15), we can obtain $G(x)=g_{0} e^{\gamma x}$. Hence, (31) leads to

$$
p(x)=\frac{1}{g_{0}}\left(G(x)+\int_{0}^{x} l(x, y) G(y) d y\right) .
$$

On the other hand, due to the invertibility of the transformation (12), we have

$$
\bar{g}(x)=G(x)+\int_{0}^{x} l(x, y) G(y) d y,
$$

where the inverse kernel $l$ is defined by (17). Thus, combining (43) and (44) yields $g_{0} p(x)=\bar{g}(x)$. Noting (3), we have $p(1) \neq 0$ if and only if $\bar{g}(1) \neq 0$.

We note that $\bar{g}(x)=g(x) \exp \int_{0}^{x} \lambda(\eta) d \eta$. Thus, $\bar{g}(1) \neq 0$ is equivalent to $g(1) \neq 0$. Then, from Proposition 2 , we have the following proposition.

Proposition 3. $p(1) \neq 0$ if and only if $g(1) \neq 0$, where $g$ is the solution of (2).

\section{Main Result}

Theorem 4. Assume that $\bar{g}(1) \neq 0$. Then, for any $u_{0} \in$ $L^{2}(0,1)$, the solution of the first-order hyperbolic system (5) with $u(\cdot, 0)=u_{0}(\cdot)$ satisfies

$$
u(x, t)=0 \text { for any } t \geq 1, x \in[0,1],
$$

where

$$
\begin{aligned}
U(t)= & -\frac{1}{\bar{g}(1)} \\
& \times\left\{\int_{0}^{1}\left[k_{x}(1, y)-\gamma k(1, y)+\bar{f}(1, y)\right] u(y, t) d y\right. \\
& +\bar{h}(1) u(0, t)\}
\end{aligned}
$$

and $\bar{g}$ is the solution of (7) with $g_{0} \neq 0$.

Under the feedback control law given in Theorem 4, the system (5) becomes

$$
\begin{gathered}
u_{t}(x, t)=u_{x}(x, t)+\left[\bar{h}(x)-\frac{\bar{g}(x)}{\bar{g}(1)} \bar{h}(1)\right] u(0, t) \\
+\int_{0}^{x} \bar{f}(x, y) u(y, t) d y \\
-\frac{\bar{g}(x)}{\bar{g}(1)} \int_{0}^{1}\left(k_{x}(1, y)-\gamma k(1, y)\right. \\
+\bar{f}(1, y)) u(y, t) d y, \\
u(1, t)=0, \quad t>0 .
\end{gathered}
$$

We define a linear operator $A$ in $L^{2}(0,1)$ as

$$
\begin{aligned}
A z= & z^{\prime}(x)+\left[\bar{h}(x)-\frac{\bar{g}(x)}{\bar{g}(1)} \bar{h}(1)\right] z(0) \\
& +\int_{0}^{x} \bar{f}(x, y) z(y) d y \\
& -\frac{\bar{g}(x)}{\bar{g}(1)} \int_{0}^{1}\left(k_{x}(1, y)-\gamma k(1, y)+\bar{f}(1, y)\right) z(y) d y,
\end{aligned}
$$

for any $z \in \mathscr{D}(A)$, where $\mathscr{D}(A)=\left\{z \in H^{1}(0,1) \mid z(1)=0\right\}$. To prove Theorem 4 , we need the following lemma.

Lemma 5. Assume that $\bar{g}(1) \neq 0$. The linear operator $A$ generates a $C_{0}$-semigroup $S(t), t \geq 0$ on $L^{2}(0,1)$.

Proof. Define another linear operator $\bar{A}$ in $L^{2}(0,1)$ by

$$
\bar{A} z=z^{\prime}(x)+\left[\bar{h}(x)-\frac{\bar{g}(x)}{\bar{g}(1)} \bar{h}(1)\right] z(0), \quad \forall z \in \mathscr{D}(\bar{A})
$$


where $\mathscr{D}(\bar{A})=\mathscr{D}(A)$. And also we define $B \in \mathscr{L}\left(L^{2}(0,1)\right)$ as

$$
\begin{array}{r}
B z=\int_{0}^{x} \bar{f}(x, y) z(y) d y-\frac{\bar{g}(x)}{\bar{g}(1)} \\
\times \int_{0}^{1}\left(k_{x}(1, y)+\gamma k(1, y)-\bar{f}(1, y)\right) z(y) d y, \\
\forall z \in L^{2}(0,1),
\end{array}
$$

where $\mathscr{L}\left(L^{2}(0,1)\right)$ denotes the set of all continuous linear operators in $L^{2}(0,1)$. Similar to the proof of Theorem 3.1 in [8], we can show that $\bar{A}$ generates a $C_{0}$-semigroup on $L^{2}(0,1)$. Moreover, since $B \in \mathscr{L}\left(L^{2}(0,1)\right)$, by the perturbation theory of semigroups (see Theorem 3.1.1 in [27]), $A=\bar{A}+B$ can generate a $C_{0}$-semigroup $S(t)(t \geq 0)$ on $L^{2}(0,1)$.

Remark 6. By Lemma 5 and the standard semigroup theory (see [27]), the system (47) has a unique mild solution $u(\cdot, t)=$ $S(t) u_{0} \in C\left([0, \infty), L^{2}(0,1)\right)$ for $u_{0} \in L^{2}(0,1)$, satisfying the initial condition $u(x, 0)=u_{0}(x)$. Moreover, if $u_{0} \in \mathscr{D}(A)$, we have $u \in C([0, \infty), \mathscr{D}(A)) \bigcap C^{1}\left([0, \infty), L^{2}(0,1)\right)$.

Remark 7. If (47) has a classical solution $u$, that is, $u$ is continuously differentiable and satisfies (47) for every $t$ and $x$, then the classical solution is the same as the mild solution, which is mentioned in Remark 6.

Proof (Theorem 4). Let $\Pi \in \mathscr{L}\left(L^{2}(0,1)\right)$ denote the backstepping transformation and let $\Pi^{-1}$ denote its inverse transformation. Since $\bar{g}(1) \neq 0$, by Proposition 2 , we have $p(1) \neq 0$. Then, it follows from Theorem 1 that $T^{-1}$ exists. Define

$$
\begin{gathered}
\mathscr{P}=\left\{\phi \in C^{1}([0,1]) \mid \phi(1)=0, \phi^{\prime}(1)=0\right\}, \quad \mathscr{V}=T^{-1} \mathscr{P}, \\
\mathscr{U}=\Pi^{-1} \mathscr{V} .
\end{gathered}
$$

We first show that, for any $u_{0} \in \mathcal{U}$, (45) holds. Let $v_{0}=$ $\Pi u_{0}$ and $\phi_{0}=T v_{0}$. Obviously, we have $v_{0} \in \mathscr{V}$ and $\phi_{0} \in \mathscr{P}$. If we take the state feedback control law as the following form:

$$
U(t)=-\frac{1}{\bar{g}(1)} \int_{0}^{1} l(1, y) \phi(y, t) d y,
$$

the system (28) can be converted into the following system:

$$
\begin{gathered}
\phi_{t}(x, t)=\phi_{x}(x, t), \\
(x, t) \in(0,1) \times(0, \infty), \\
\phi(1, t)=0, \quad t \in(0, \infty) .
\end{gathered}
$$

Then, for $\phi_{0} \in \mathscr{P}$, (53) has a unique solution $\phi \in C^{1}([0,1] \times$ $[0, \infty))$ taking the following form:

$$
\phi(x, t)= \begin{cases}\phi_{0}(x+t), & 0 \leq x+t<1 \\ 0, & x+t \geq 1\end{cases}
$$

where $\phi(x, 0)=\phi_{0}(x)$ is the initial condition.
Let $v(\cdot, t)=T^{-1} \phi(\cdot, t)$ for $t \geq 0$. Making use of (33) and (37) yields

$$
\begin{aligned}
v(x, t)= & -\frac{e^{\gamma x}}{p(1)} \int_{0}^{1} \phi(y, t) \Gamma(y) d y \\
& +\int_{0}^{x} e^{\gamma(x-y)} \phi(y, t) d y,
\end{aligned}
$$

from which and the property of $\phi$ we can see easily $v \in$ $C^{1}([0,1] \times[0, \infty))$. Moreover, we can even show that $v$ is twice continuously differentiable; that is, $v \in C^{2}([0,1] \times[0, \infty))$. In fact, directly taking derivatives of (55) yields that

$$
\begin{gathered}
v_{x x} \in C([0,1] \times[0, \infty)), \\
v_{x t}=x_{t x} \in C([0,1] \times[0, \infty)),
\end{gathered}
$$

where we note $\phi \in C^{1}([0,1] \times[0, \infty))$. Since, by (53),

$$
\begin{aligned}
v_{t}= & -\frac{e^{\gamma x}}{p(1)} \int_{0}^{1} \phi_{t}(y, t) \Gamma(y) d y+\int_{0}^{x} e^{\gamma(x-y)} \phi_{t}(y, t) d y \\
= & -\frac{e^{\gamma x}}{p(1)} \int_{0}^{1} \phi_{y}(y, t) \Gamma(y) d y+\int_{0}^{x} e^{\gamma(x-y)} \phi_{y}(y, t) d y \\
= & -\left.\frac{e^{\gamma x}}{p(1)} \phi(y, t) \Gamma(y)\right|_{y=0} ^{y=1}+\frac{e^{\gamma x}}{p(1)} \int_{0}^{1} \phi(y, t) \Gamma^{\prime}(y) d y \\
& +\left.e^{\gamma(x-y)} \phi(y, t)\right|_{y=0} ^{y=x}+\gamma \int_{0}^{x} e^{\gamma(x-y)} \phi(y, t) d y \\
= & \frac{e^{\gamma x}}{p(1)} \int_{0}^{1} \phi(y, t) \Gamma^{\prime}(y) d y+\phi(x, t) \\
& +\gamma \int_{0}^{x} e^{\gamma(x-y)} \phi(y, t) d y,
\end{aligned}
$$

where we use the facts that $\Gamma(0)=p(1)$ and $\phi(1, t)=0$, then,

$$
\begin{aligned}
v_{t t}= & \frac{e^{\gamma x}}{p(1)} \int_{0}^{1} \phi_{t}(y, t) \Gamma^{\prime}(y) d y+\phi_{t}(x, t) \\
& +\gamma \int_{0}^{x} e^{\gamma(x-y)} \phi_{t}(y, t) d y \in C([0,1] \times[0, \infty)) .
\end{aligned}
$$

From (56) and (58), we can obtain that $v \in C^{2}([0,1] \times[0, \infty))$, which implies that the regularity assumption in Section 3.3 is satisfied. Thus, the differential transformation (20) can be applied and $v$ defined by (55) is the solution of (19) with the following feedback:

$$
U(t)=-\frac{1}{\bar{g}(1)} \int_{0}^{1} l(1, y)\left[v_{y}(y, t)-\gamma v(y, t)\right] d y,
$$

such that $v(\cdot, 0)=v_{0}(\cdot)$, where $v_{0}=T^{-1} \phi_{0} \in \mathscr{V}$. Here, the feedback (59) just comes from the feedback (52) due to the differential transformation (20). Thus, noting $\phi(x, t)=0$ for $t \geq 1, x \in[0,1]$, it follows from (55) that

$$
v(x, t)=0 \quad \text { for } t \geq 1, x \in[0,1] .
$$


To prove this theorem, we need to show that the control law (46) comes from the feedback control law (59). Let $u$ be the inverse backstepping transformation of $v$; that is, $u$ satisfies (16). From (8), (16), and (17), one can check that

$$
\begin{aligned}
& \int_{0}^{x} l(x, y) v_{y}(y, t) d y \\
&= l(x, x) v(x, t)-l(x, 0) v(0, t) \\
& \quad-\int_{0}^{x} l_{y}(x, y) v(y, t) d y \\
&= l(x, x) v(x, t) \\
&+\int_{0}^{x} l_{x}(x, y) v(y, t) d y-l(x, 0) v(0, t) \\
&+\int_{0}^{x} \int_{y}^{x} \bar{f}(x, \eta) l(\eta, y) v(y, t) d \eta d y \\
&+\int_{0}^{x} \bar{f}(x, y) v(y, t) d y \\
&= l(x, x) v(x, t) \\
&+\int_{0}^{x} l_{x}(x, y) v(y, t) d y-l(x, 0) v(0, t) \\
&+\int_{0}^{x} \bar{f}(x, y)\left[v(y, t)+\int_{0}^{y} l(y, \eta) v(\eta, t) d \eta\right] d y \\
&= u_{x}(x, t)-v_{x}(x, t)-l(x, 0) v(0, t) \\
&+\int_{0}^{x} \bar{f}(x, y) u(y, t) d y \\
&= k(x, x) u(x, t) \\
&+\int_{0}^{x}\left[k_{x}(x, y)+\bar{f}(x, y)\right] u(y, t) d y+\bar{h}(x) u(0, t), \\
& \int_{0}^{x} l(x, y) v(y, t) d y=u(x, t)-v(x, t) \\
& \quad
\end{aligned}
$$

Thus, combining (59) with (61) leads to

$$
\begin{aligned}
U(t)= & -\frac{1}{\bar{g}(1)} \\
& \times\left\{\int_{0}^{1}\left[k_{x}(1, y)-\gamma k(1, y)+\bar{f}(1, y)\right]\right. \\
& \quad \times u(y, t) d y+\bar{h}(1) u(0, t)\},
\end{aligned}
$$

where we have used the fact $u(1, t)=0$. Then, for $u_{0}=$ $\Pi^{-1} v_{0} \in \mathcal{U}, u(x, t)$ defined by (16) is the solution of (5) with the feedback (46) and $u(\cdot, 0)=u_{0}(\cdot)$. Moreover, by $l \in C^{1}([0,1] \times[0,1])$ and $v \in C^{1}([0,1] \times[0, \infty))$, we have $u \in C^{1}([0,1] \times[0, \infty))$. Thus, it follows from (16) and (60) that for any $u_{0} \in \mathscr{U}$

$$
u(x, t)=0 \quad \text { for } t \geq 1, x \in[0,1] .
$$

Now, we will show that, for any $u_{0} \in L^{2}(0,1)$, (45) holds. By Remark 6, we have that, for any $u_{0} \in L^{2}(0,1)$, $u(x, t)=S(t) u_{0}$ is the mild solution of system (47), that is, the mild solution of (5) with the feedback (46), where $S(t)$ is the solution semigroup of (47). Then, by (63), we only need to prove that $\mathcal{U}$ is dense in $L^{2}(0,1)$. Since $A$ is a generator of $C_{0}$ semigroup of $S(t)$, we have $\mathscr{D}(A)$ is dense in $L^{2}(0,1)$. Thus, for any $u_{0} \in L^{2}(0,1)$ and $\epsilon>0$, we can find $\widetilde{u}_{0} \in \mathscr{D}(A)$ such that

$$
\left\|u_{0}-\tilde{u}_{0}\right\| \leq \frac{\epsilon}{2} .
$$

By the definition of the backstepping transformation, we have $\Pi$ is a bijection from $\mathscr{D}(A)$ to $\mathscr{D}(T)$. Then, $\Pi \widetilde{u}_{0} \in \mathscr{D}(T)$. Thus, $T \Pi \widetilde{u}_{0} \in L^{2}(0,1)$ is well defined. since $\mathscr{P}$ is dense in $L^{2}(0,1)$, there exists $\widetilde{\phi}_{0} \in \mathscr{P}$ such that

$$
\left\|T \Pi \widetilde{\mathcal{u}}_{0}-\widetilde{\phi}_{0}\right\| \leq \frac{\epsilon}{2\left\|T^{-1}\right\|\left\|\Pi^{-1}\right\|} .
$$

Then, we have $\Pi^{-1} T^{-1} \widetilde{\phi}_{0} \in \mathcal{U}$ and, by (64),

$$
\begin{aligned}
& \left\|u_{0}-\Pi^{-1} T^{-1} \widetilde{\phi}_{0}\right\| \\
& \quad \leq\left\|u_{0}-\widetilde{u}_{0}\right\|+\left\|\widetilde{u}_{0}-\Pi^{-1} T^{-1} \widetilde{\phi}_{0}\right\| \\
& \leq\left\|u_{0}-\widetilde{u}_{0}\right\|+\left\|\Pi^{-1}\right\|\left\|T^{-1}\right\|\left\|T \Pi \widetilde{u}_{0}-\widetilde{\phi}_{0}\right\| \\
& \quad \leq \epsilon,
\end{aligned}
$$

which implies that $\mathscr{U}$ is a dense subset in $L^{2}(0,1)$. Thus, we complete the proof of Theorem 4 .

By making use of the transformations (4) and (6), we can obtain the following theorem based on Theorem 4 .

Theorem 8. Assume that $g(1) \neq 0$. Then, for any $w_{0} \in$ $L^{2}(0,1)$, the solution of the first-order hyperbolic system (1) with $w(\cdot, 0)=w_{0}(\cdot)$ satisfies

$$
w(x, t)=0 \quad \text { for any } t \geq 1, x \in[0,1],
$$

where

$$
\begin{aligned}
U(t)=-\frac{1}{g(1)} \int_{0}^{1}\left[k_{x}(1, y)-\gamma k(1, y)\right] \\
\quad \times \exp \left\{-\int_{y}^{1} \lambda(\eta) d \eta\right\} w(y, t) d y \\
-\frac{1}{g(1)} \int_{0}^{1} f(1, y) w(y, t) d y-\frac{h(1)}{g(1)} w(0, t)
\end{aligned}
$$

and $g$ is the solution of (2) with $g_{0} \neq 0$. 
Remark 9. For any given $g_{0} \neq 0$, to guarantee the existence of the feedback control law (46), we only need to design the parameter $\gamma$ in $(7)$ such that $\bar{g}(1) \neq 0$. Equivalently, we can design the parameter $\gamma$ in (2) such that $g(1) \neq 0$, which ensures the existence of the feedback control law (68).

Remark 10. Let $\gamma$ be given. For two different $g_{0}^{i}, i=1,2$, let $g^{i}$ be the solutions of (2) by taking $g_{0}=g_{0}^{i}$, respectively. Moreover, for each $i=1,2$, let $U^{i}$ be the corresponding temporal component of the feedback law. Then one can see that if $g^{i}(1) \neq 0, i=1,2$, it follows from $(2)$ that $g^{1}(x) / g^{1}(1)=$ $g^{2}(x) / g^{2}(1)$. Thus, the two interior actuation functions satisfy $g^{1}(x) U^{1}(t)=g^{2}(x) U^{2}(t)$ for $x \in[0,1], t \geq 0$.

\section{Numerical Simulation}

Now we consider the following 1-dimensional hyperbolic PDE (i.e., by taking $\lambda(x)=a, h(x)=0, f(x, y)=p e^{q(x-y)}$ in (1)):

$$
\begin{aligned}
w_{t}(x, t)= & w_{x}(x, t)+a w(x, t) \\
& +\int_{0}^{x} p e^{q(x-y)} w(y, t) d y+g(x) U(t), \\
& w(1, t)=0, \quad t \in(0, \infty),
\end{aligned}
$$

where $a, p, q$ are constants. Then the kernel equation (9) becomes

$$
\begin{gathered}
k_{x}(x, y)+k_{y}(x, y) \\
=p \int_{y}^{x} k(x, \eta) e^{(a+q)(\eta-y)} d \eta-p e^{(a+q)(x-y)}, \\
k(x, 0)=0 .
\end{gathered}
$$

One can get the closed-from solution of (70) (see Example 2.2 in [7])

$$
k(x, y)=-p e^{(a+q)(x-y)} y \frac{I_{1}(2 \sqrt{x(x-y)})}{\sqrt{x(x-y)}},
$$

where $I_{1}$ is the modified Bessel functions of order one.

We choose $a=2, p=2$, and $q=0.5$ in (69) and set the initial condition as $w(x, 0)=\sin (2 \pi x)$. From Figure 1 , we can observe that the system is unstable from the numerical result which is obtained using the finite difference method. The integral term in the PDE system (69) is the main reason to cause instability of the open-loop system. Thus, a feedback control law is necessary to achieve closed-loop stability. The interior actuation function includes two components, that is, the temporal function $U(t)$ and the spatial shape function $g(x)$. The temporal component $U(t)$ is given by (68) and the spatial shape function $g(x)$ is given by the ODE (2) with the design parameter $\gamma=4.5$. By Remark 9, without loss of generality, we take

$$
g_{0}=1
$$

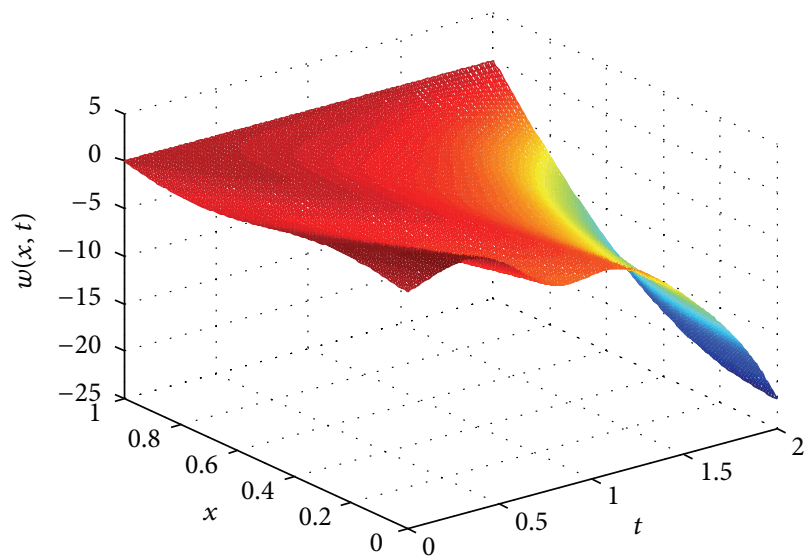

Figure 1: The response of the open-loop system.

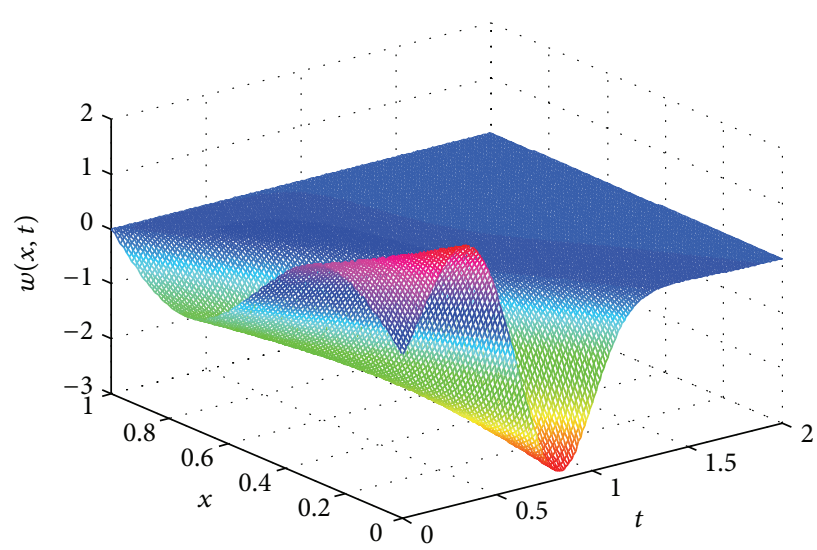

FIgURE 2: The response of the closed-loop system $(\gamma=4.5)$.

in (2). For the closed-loop simulation, we solve the PDE system (69) with $U(t)$ and $g(x)$ given in Theorem 8 using the finite difference method. The response of the closed-loop system is shown in Figure 2. In addition, the corresponding temporal function $U(t)$ in the closed-loop simulation is shown in Figure 3. The result in Theorem 8 states that the closed-loop system should satisfy $w(x, t)=0$ for any $t \geq 1$ and $x \in[0,1]$. As shown in Figures 2 and 3, one may realize that the state trajectory $w$ and the boundary trajectory $U(t)$ are close to but do not exactly reach zero, which is completely due to the unavoidable numerical errors. By improving the numerical accuracy, one may take the values closer to zero at $t=1$, which implies that the numerical results validate the conclusion in Theorem 8. The shape function $g(x)$ is shown in Figure 4 which is a monotonically increasing function over $x \in[0,1]$. The interior actuation, that is, the multiplication term $g(x) U(t)$, is shown in Figure 5.

By Theorem 8 , the constant $\gamma$ in (2) is a parameter to be designed such that $g(1) \neq 0$. For every such $\gamma$, there exist $g$ and $U$ that can stabilize the unstable system. A natural problem is which the optimal parameter is in some proper sense. By varying $\gamma$ in the internal $[-15,5]$, we can solve the ODE (2) for $g(1)$ numerically and the result is shown in Figure 6. We find that, for $\gamma<2.5, g(1)$ is close to 


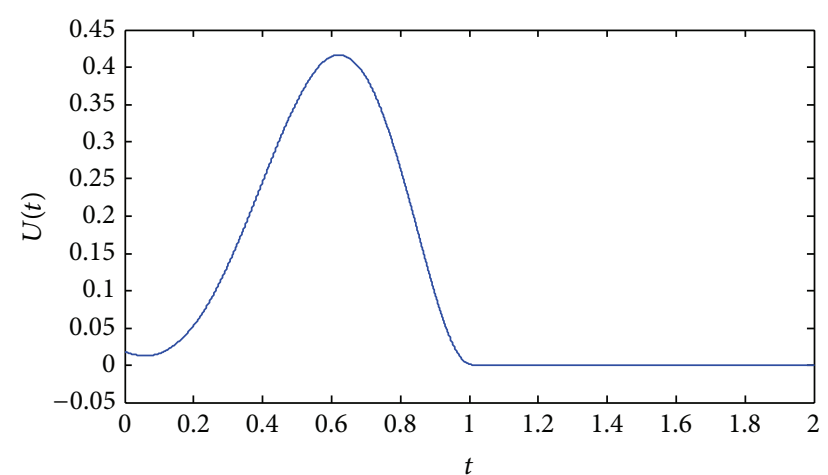

FIgURE 3: The temporal function $U(t)(\gamma=4.5)$.

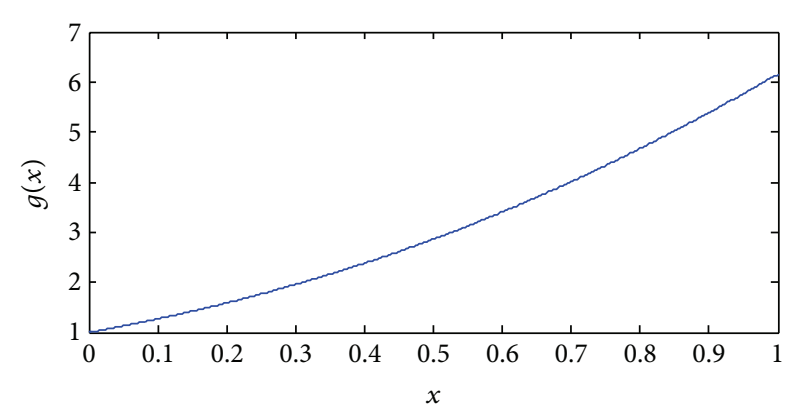

FIGURE 4: The shape function $g(x)(\gamma=4.5)$.

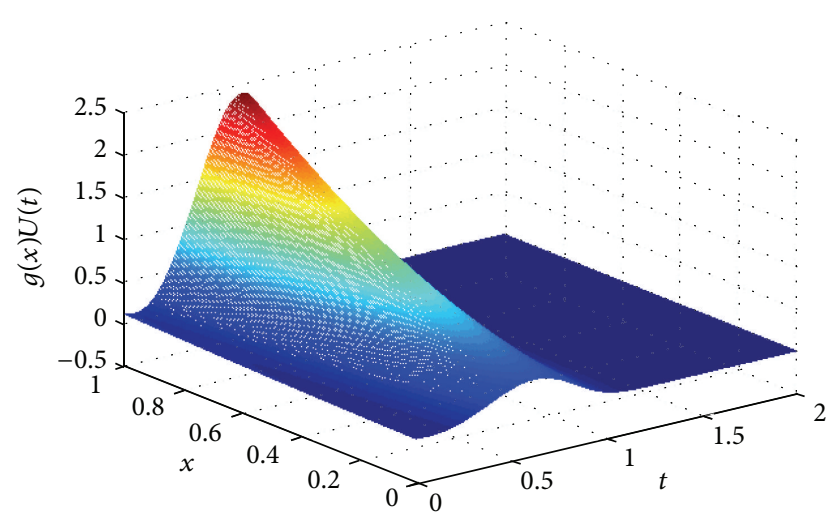

FIGURE 5: The interior actuation function $g(x) U(t)(\gamma=4.5)$.

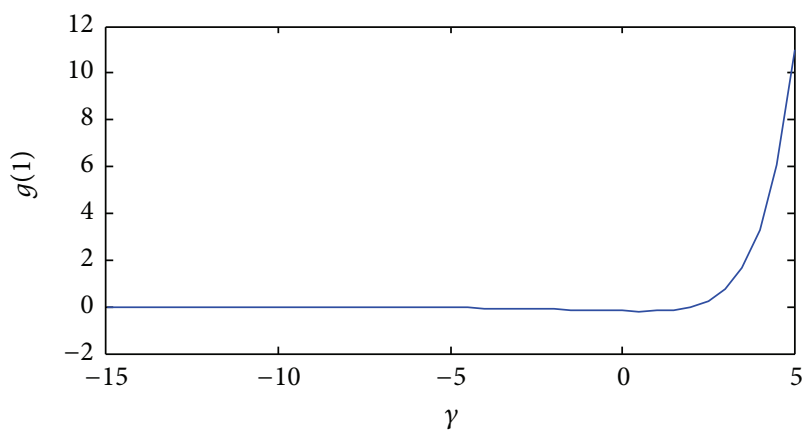

Figure 6: $g(1)$ for different $\gamma(\gamma \in[2.5,10])$.

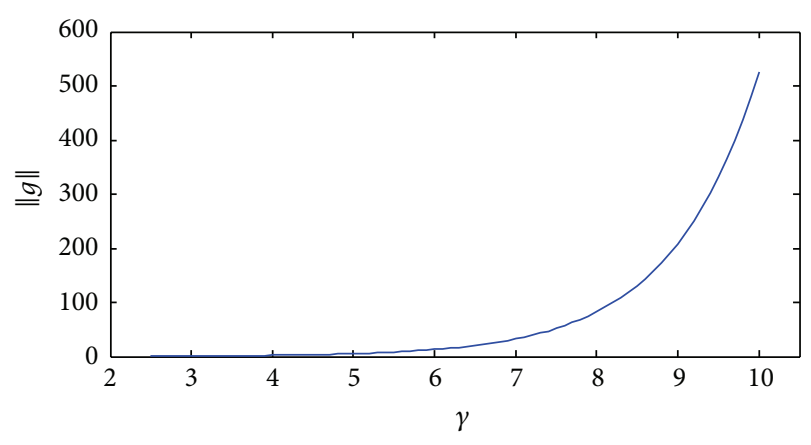

Figure 7: The $L_{2}$-norm of $g$ for different $\gamma(\gamma \in[2.5,10],\|g\|=$ $\left.\left(\int_{0}^{1}|g(x)|^{2} d x\right)^{1 / 2}\right)$.

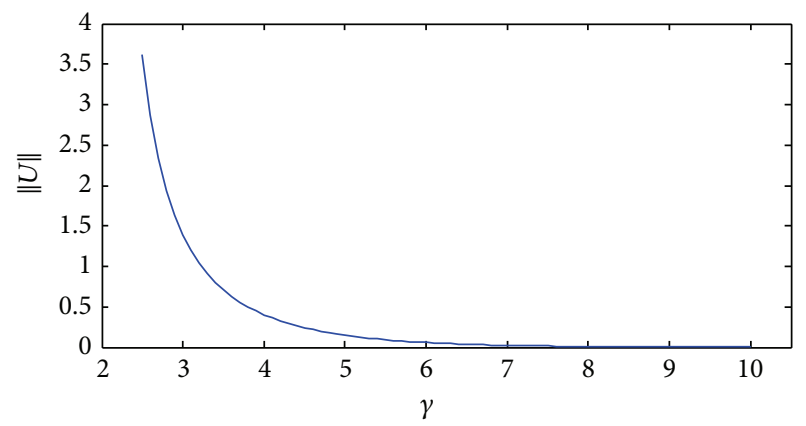

FIGURE 8: The $L_{2}$-norm of $U$ for different $\gamma(\gamma \in[2.5,10],\|U\|=$ $\left.\left(\int_{0}^{T}|U(t)|^{2} d t\right)^{1 / 2}, T=2\right)$.

zero (as shown in Figure 6). In order to avoid numerical singularities, we need to choose $\gamma$ properly such that $g(1)$ is obviously different from zero. For this reason, we will discuss the case for $\gamma \geq 2.5$. We compute $g(x)$ and its $L_{2}$-norm for $\gamma \in[2.5,10]$. One can observe in Figure 7 that the $L_{2}-$ norm is increasing dramatically as the value of $\gamma$ increases. Similarly, we also compute $U(t)$ and its $L_{2}$-norm for $\gamma \in$ $[2.5,10]$, shown in Figure 8 . We note that the $L_{2}$-norm is a monotonically decreasing function of $\gamma$. It is shown in Figure 9 that the $L_{2}$-norm of $g(x) U(t)$ varies with respect to $\gamma$ and we find that there exists a point $\gamma=5.9$, such that the $L_{2}$-norm of the control function $g U$ is minimized $\left(\min _{\gamma \in[2.5,10]}\|g U\|=0.76\right)$.

We can realize that the spatial-temporal actuation $g(x) U(t)$ is not practically applicable in real world applications since it requires external forcing over the whole physical domain, that is, $x \in[0,1]$. Motivated by the subdomain actuation mechanism in engineering practice, a more practical problem can be formulated as follows, associated with the simulation model in (69):

$$
\begin{aligned}
w_{t}(x, t)= & w_{x}(x, t)+a w(x, t) \\
& +\int_{0}^{x} p e^{q(x-y)} w(y, t) d y+\chi_{l}(x) g(x) U(t), \\
& w(1, t)=0, \quad t \in(0, \infty),
\end{aligned}
$$




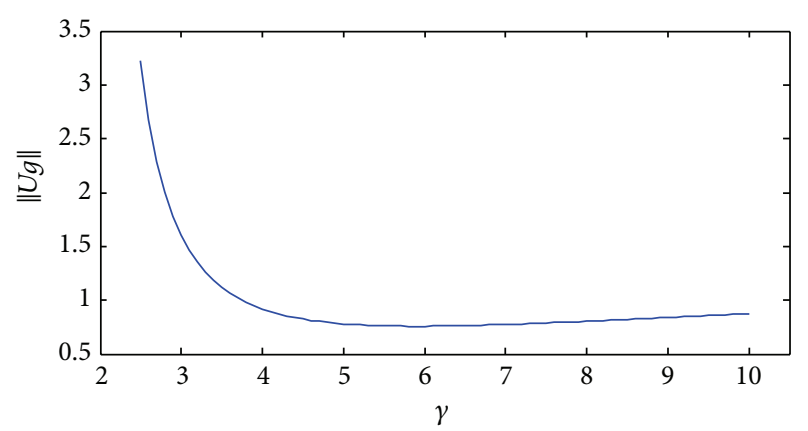

FIGURE 9: The $L_{2}$-norm of $U g$ for different $\gamma(\gamma \in[2.5,10]$, $\|U g\|=$ $\left.\left(\int_{0}^{1} \int_{0}^{T}|U(t) g(x)|^{2} d t d x\right)^{1 / 2}, T=2\right)$.

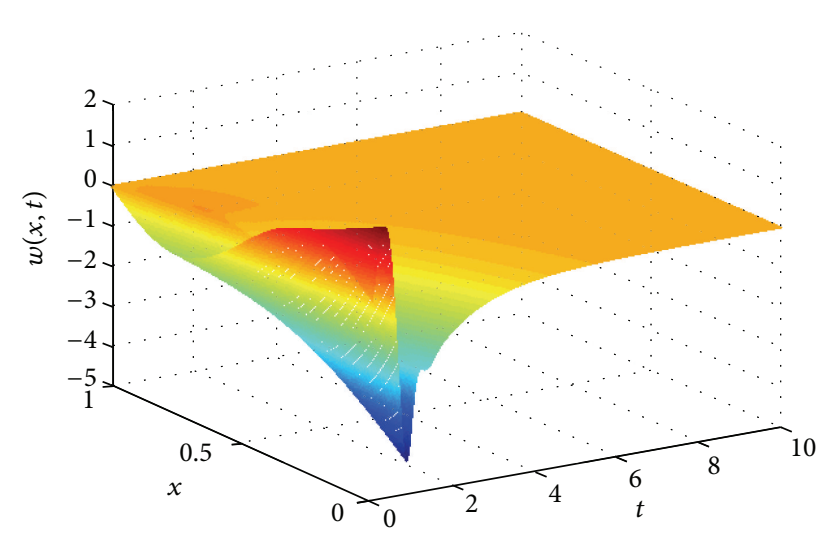

FIGURE 10: The response of the closed-loop system control acting on $[0.7,1](\gamma=4.5)$.

where $0<l<1$ and $\chi_{l}$ is the characteristic function of $[l, 1]$; that is,

$$
\chi_{l}(x)= \begin{cases}1, & x \in[l, 1], \\ 0, & x \in[0, l) .\end{cases}
$$

Given $g(x)$ and $U(t)$ being synthesized based on Theorem 8, then we use only numerical simulation rather than complete theoretical analysis in the current work to validate the effectiveness of the feedback controller even for the subdomain actuation in practical applications.

As shown in Figure 10, for $\gamma=4.5$, when the feedback control acts only on the subdomain $[0.7,1]$, the system $(73)$ can be also stabilized by only using the controller which is synthesized for the spatial-temporal actuation. But the closed-loop system takes much longer to dissipate zero at $t=7$ instead of $t=1$ for the spatial-temporal actuation (see Figure 2). When we decrease the acting domain area further to $[0.8,1]$, then the system $(73)$ can not be stabilized (see Figure 11). However, if we increase the value of $\gamma$ from $\gamma=4.5$ to $\gamma=7$, then the closed-loop system (73) appears to be stabilized again (see Figure 12). This is a quite interesting phenomenon and we try to give an explanation from Figure 13 where we solve $g(x)$ for different values of $\gamma$. One can readily find in the simulation that $g(x)$ increase dramatically as $\gamma$ does. For the characteristic function $\chi_{[0,8,1]}$ the controller is not capable of stabilizing the systems for $\gamma=4.5$. But,

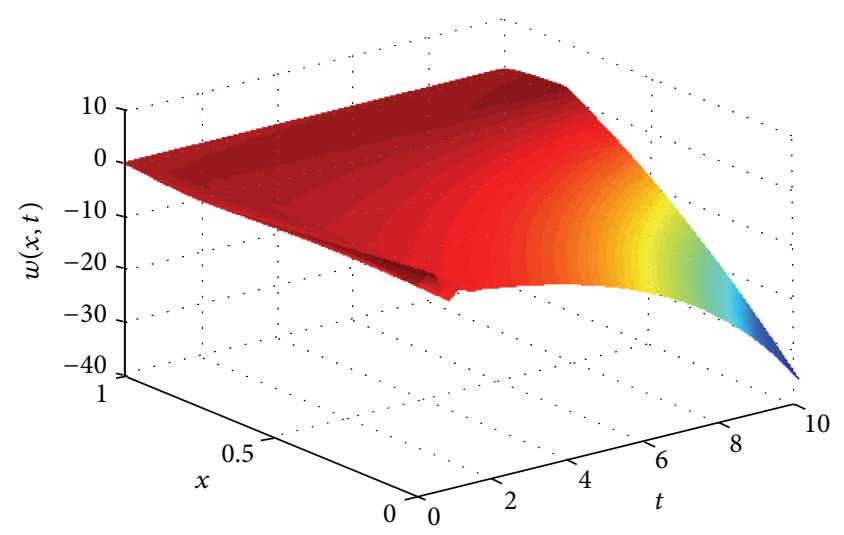

FIgURE 11: The response of the closed-loop system with control acting on $[0.8,1](\gamma=4.5)$.

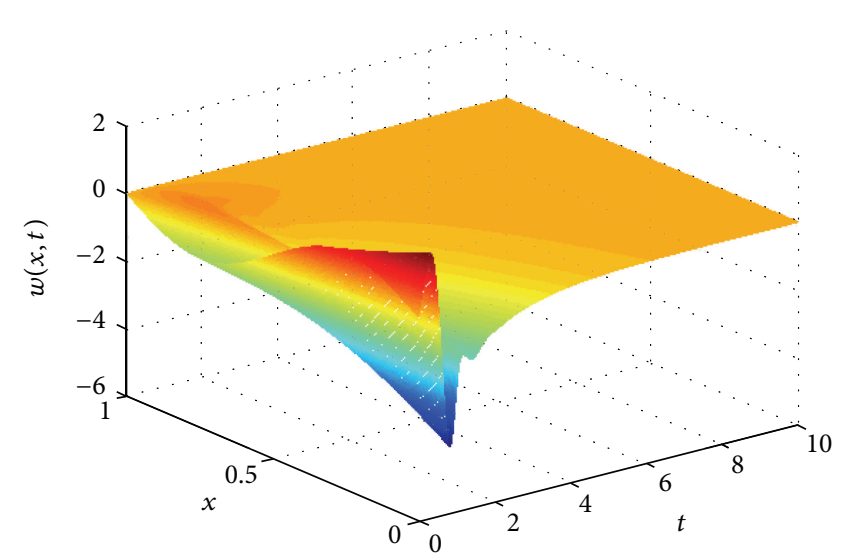

FIgURE 12: The response of the closed-loop system with control acting on $[0.8,1](\gamma=7)$.

by increasing the value of $\gamma$, it is an equivalent approach to improve the actuation strength over the subdomain close to $x=1$, where the spatial component $g(x)$ is much more significant than the values taken close to $x=0$. Alternatively, one can conclude that subdomains, where $g(x)$ can achieve significant values, are better locations to deploy actuation. A thorough theoretical analysis is definitely needed in the future.

\section{Conclusions}

In this paper, the stabilization problem using backstepping techniques for first-order hyperbolic PDEs with internal actuation has been investigated. For this, backstepping integral transformations together with a differential transformation are used to determine the state feedback controller. In future work, several directions can be pursued. First, just as said in Section 6, the theoretical study on the design of the parameter $\gamma$ is needed. Second, with this work done, we may expect to extend this paper to various PDE controlled systems arising in applied physics and engineering. Finally, the stabilization problem with internal actuation for nonlinear systems can be investigated. 


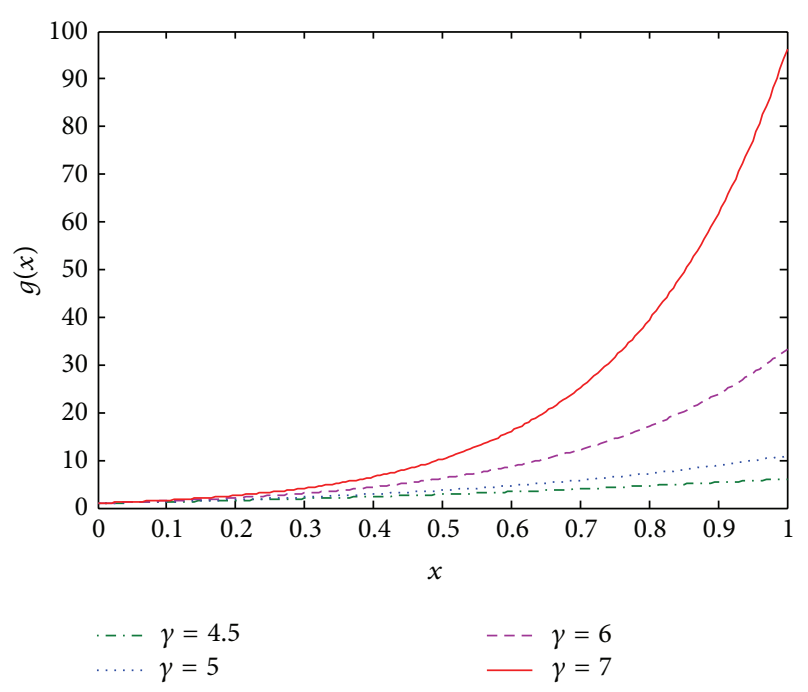

Figure 13: The shape function $g(x)$ for different $\gamma(\gamma=4.5,5,6,7)$.

\section{Conflict of Interests}

The authors declare that there is no conflict of interests regarding the publication of this paper.

\section{Acknowledgments}

This work was partially supported by the National Natural Science Foundation of China (61374096 and 61104048) and the Natural Science Foundation of Zhejiang (Y6110751).

\section{References}

[1] A. Bensoussan, G. da Prato, M. Delfour, and S. K. Mitter, Representation and Control of Infinite Dimensional Systems, Birkhäuser, 2nd edition, 2007.

[2] R. F. Curtain and H. Zwart, An Introduction to In finiteDimensional Linear Systems Theory, Springer, 1995.

[3] R. Glowinski, J. Lions, and J. He, Exact and Approximate Controllability for Distributed Parameter Systems: A Numerical Approach, Cambridge University Press, 2007.

[4] I. Lasiecka and R. Triggiani, Control Theory for Partial Differential Equations, Cambridge University Press, Cambridge, UK, 2000 .

[5] Z. Luo, B. Guo, and O. Morgul, Stability and Stabilization of Infinite Dimensional Systems with Applications, Communications and Control Engineering, Springer, London, UK, 1999.

[6] J. Coron, B. d'Andrea Novel, and G. Bastin, "A strict Lyapunov function for boundary control of hyperbolic systems of conservation laws," IEEE Transactions on Automatic Control, vol. 52, no. 1, pp. 2-11, 2007.

[7] M. Krstic and A. Smyshlyaev, "Backstepping boundary control for first-order hyperbolic PDEs and application to systems with actuator and sensor delays," Systems and Control Letters, vol. 57, no. 9, pp. 750-758, 2008.
[8] H. Sano, "Exponential stability of a mono-tubular heat exchanger equation with output feedback," Systems and Control Letters, vol. 50, no. 5, pp. 363-369, 2003.

[9] X. Yu and K. Liu, "Eventual regularity of the semigroup associated with the mono-tubular heat exchanger equation with output feedback," Systems and Control Letters, vol. 55, no. 10, pp. 859-862, 2006.

[10] M. Krstic, I. Kanellakopoulos, and P. Kokotovic, Nonlinear and Adaptive Control Design, John Wiley and Sons, New York, NY, USA, 1995.

[11] J. Coron and B. d'Andrea-Novel, "Stabilization of a rotating body beam without damping," IEEE Transactions on Automatic Control, vol. 43, no. 5, pp. 608-618, 1998.

[12] M. Krstic, B. Guo, A. Balogh, and A. Smyshlyaev, "Outputfeedback stabilization of an unstable wave equation," Automatica, vol. 44, no. 1, pp. 63-74, 2008.

[13] M. Krstic and A. Smyshlyaev, Boundary Control of PDEs. A Course on Backstepping Designs, Society for Industrial and Applied Mathematics, 2008.

[14] W. Liu, "Boundary feedback stabilization of an unstable heat equation," SIAM Journal on Control and Optimization, vol. 42, no. 3, pp. 1033-1043, 2003.

[15] T. Meurer and A. Kugi, "Tracking control for boundary controlled parabolic PDEs with varying parameters: combining backstepping and differential flatness," Automatica, vol. 45, no. 5, pp. 1182-1194, 2009.

[16] C. Xu, E. Schuster, R. Vazquez, and M. Krstic, "Stabilization of linearized 2D magnetohydrodynamic channel flow by backstepping boundary control," Systems \& Control Letters, vol. 57, no. 10, pp. 805-812, 2008.

[17] R. Vazquez and M. Krstic, "A closed-form feedback controller for stabilization of the linearized 2-D Navier-Stokes poiseuille system," IEEE Transactions on Automatic Control, vol. 52, no. 12, pp. 2298-2312, 2007.

[18] R. Vazquez, E. Schuster, and M. Krstic, "Magnetohydrodynamic state estimation with boundary sensors," Automatica, vol. 44, no. 10, pp. 2517-2527, 2008.

[19] O. M. Aamo, "Disturbance rejection in $2 \times 2$ linear hyperbolic systems," IEEE Transactions on Automatic Control, vol. 58, no. 5, pp. 1095-1106, 2013.

[20] T. Meurer, Control of Higher-Dimensional PDEs: Flatness and Backstepping Designs, Springer, New York, NY, USA, 2013.

[21] E. Cerpa and J. Coron, "Rapid stabilization for a Kortewegde Vries equation from the left Dirichlet boundary condition," IEEE Transactions on Automatic Control, vol. 58, no. 7, pp. 16881695, 2013.

[22] J. Coron, R. Vazquez, M. Krstic, and G. Bastin, "Local exponential $H^{2}$ stabilization of a $2 \times 2$ quasilinear hyperbolic system using backstepping," SIAM Journal on Control and Optimization, vol. 51, no. 3, pp. 2005-2035, 2013.

[23] I. Lasiecka and R. Triggiani, "The regulator problem for parabolic equations with Dirichlet boundary control. I. Riccati's feedback synthesis and regularity of optimal solution," Applied Mathematics and Optimization, vol. 16, no. 2, pp. 147-168, 1987.

[24] I. Lasiecka and R. Triggiani, "The regulator problem for parabolic equations with Dirichlet boundary control. II. Galerkin approximation," Applied Mathematics and Optimization, vol. 16, no. 3, pp. 187-216, 1987. 
[25] W. Liu, Feedback Stabilization of the Linear ReactionConvection-Diffusion Equation and the Wave Equation, Springer, New York, NY, USA, 2009.

[26] D. Tsubakino, M. Krstic, and S. Hara, "Backstepping control for parabolic PDEs with in-domain actuation," in Proceedings of the American Control Conference (ACC '12), pp. 2226-2231, June 2012.

[27] A. Pazy, Semigroups of Linear Operators and Applications to Partial Differential Equations, vol. 44 of Applied Mathematical Sciences, Springer, New York, NY, USA, 1983. 


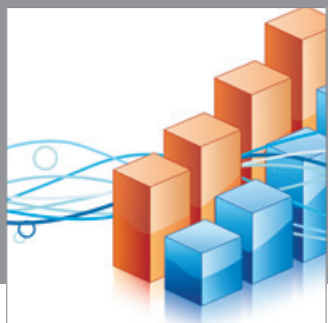

Advances in

Operations Research

mansans

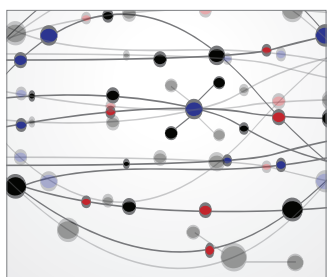

The Scientific World Journal
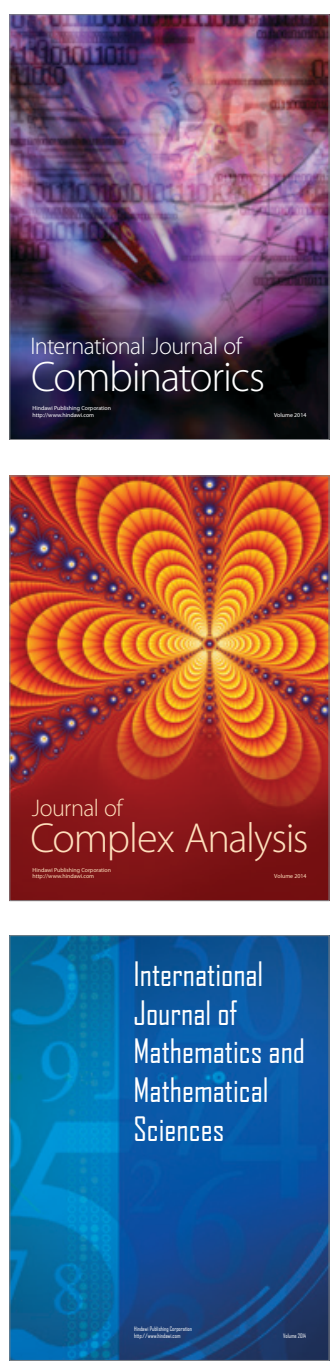
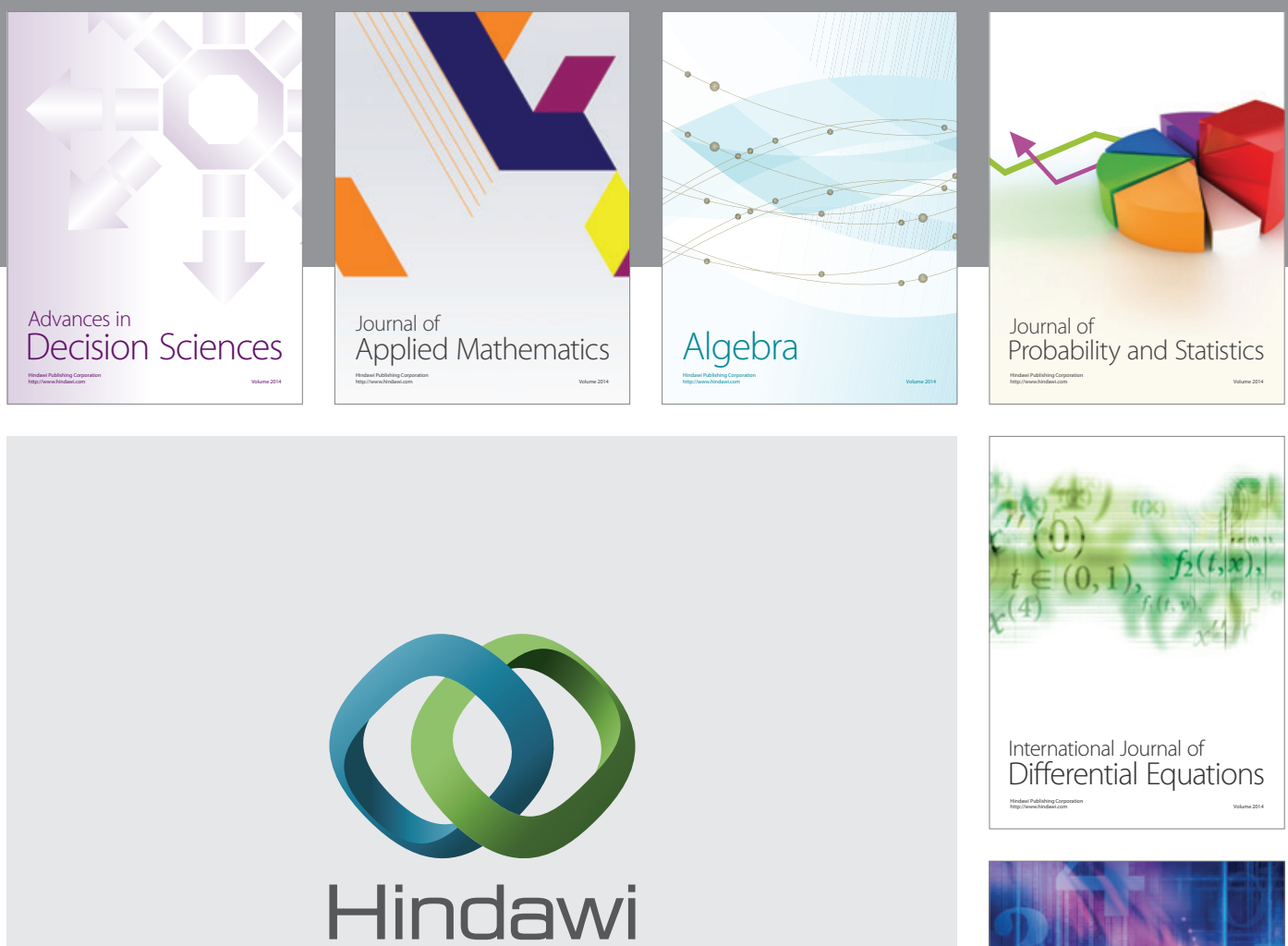

Submit your manuscripts at http://www.hindawi.com
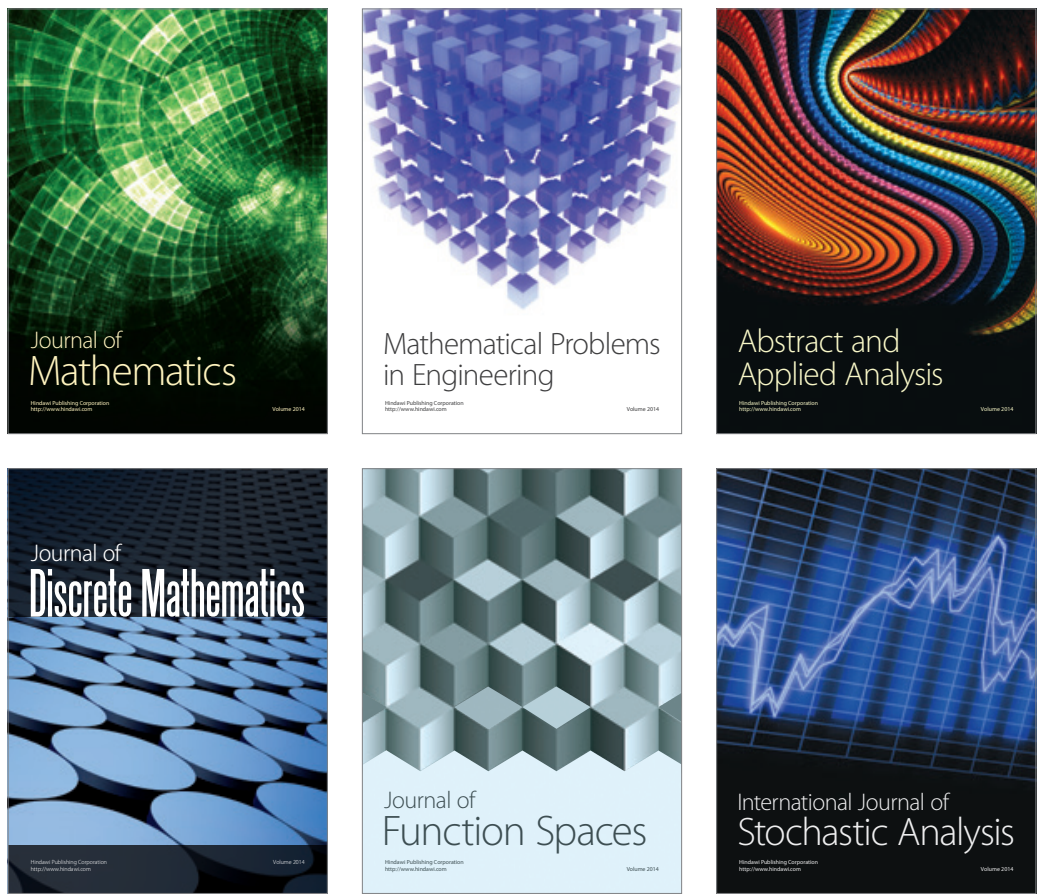

Journal of

Function Spaces

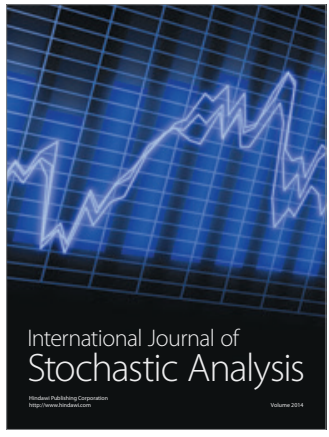

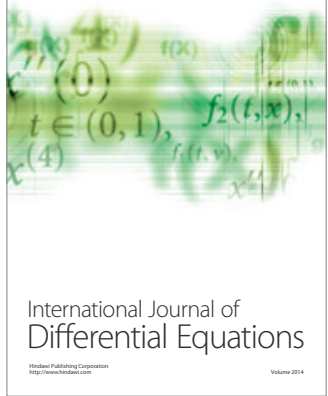
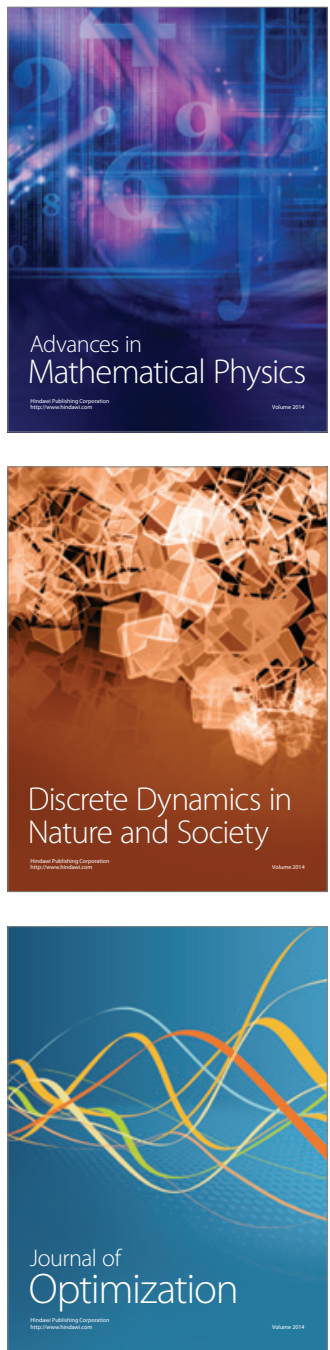